International Journal of Linguistics, Literature and Translation

ISSN: 2617-0299 (Online); ISSN: 2708-0099 (Print)

DOI: $10.32996 / \mathrm{ijllt}$

Journal Homepage: www.al-kindipublisher.com/index.php/ijltt

IJLLT

\title{
The German Orientalist School Vis-à-vis the History of Arabic Literature: Carle Brockelmann as a Locus Classicus
}

Hakeem Salmon 8 (D)

المدرسة الاستشراقية الألمانية إزاء تاريخ الأدب العربي: كارل بروكلمان نموذجاً

Lagos State University, Nigeria

$\triangle$ Corresponding Author: Hakeem Salmon, E-mail: hasbyrabby66@yahoo.com

ARTICLE INFORMATION

Received: April 08, 2021

Accepted: May 14, 2021

Volume: 4

Issue: 5

DOI: $10.32996 /$ ijllt.2021.4.5.26

\section{KEYWORDS}

Orientalism, Orientalist, Arabic literature, Islamic languages, Arab writers

\section{ABSTRACT}

It is an incontestable fact and incontrovertible truism that Orientalism- a term deployed to signify a socio-political trend signifying intellectual enquiry and the academic study of Eastern cultures by the Western intelligentsia - is one of the sources of information about Islam and Muslims. This is a culmination of gargantuan endeavours lent Arab autochthonous patrimony; whether the fragments scribbled in pure Arabic; or those documented in other Asian or African languages; or other Islamic languages such as Persia, Urdu and Turkish; in terms of preservation, study, editing, publication, or indexing. It would be pertinent here to mention the tremendous efforts the Muslims have made to follow what the Westerners have accomplished. The issue of Orientalism has polarized the Arab writers into two extremes: the Revolutionary, obsessed with an unbridled, enthusiastic penchant and infatuated with an irrational hallucinatory predilection to the level of deference and obsequiousness; and the Neo-conservative who discern it as a reprehensible scourge and pestilential plague that should not be embraced at all; not even with a long pole. Between these extremes, we have yet another constellation that is liberal, moderate and detached in its assessment of any matter with a scintilla of nexus to Orientalism. While identifying ourselves and pitching our tent with this coterie, we hereby present Carle Brockelmann, an iconic connoisseur and illustrious belletrist from German mise-en-scene (based on the application of the theory of 'Aqiq's taxonomy of Orientalists according to geographical cleavage as propounded in his Encyclopedia christened al-Istishraq wa 'I-Mustashriqun ) and dissect through analytical framework his blazing trail feat in the stratosphere of History of Arabic Literature.

الملخص:

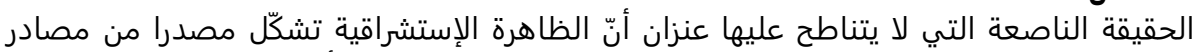

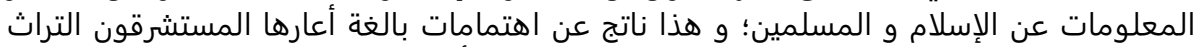

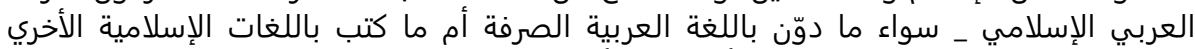

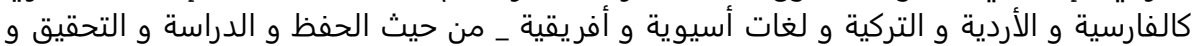

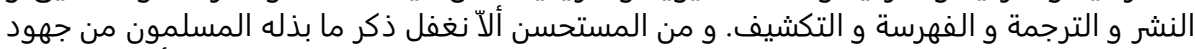

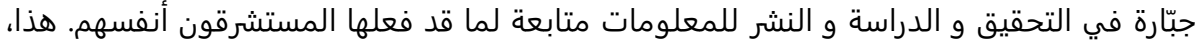

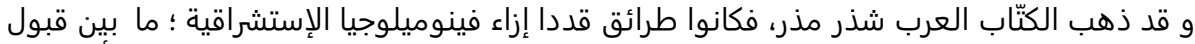

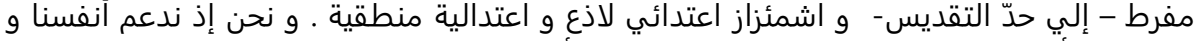

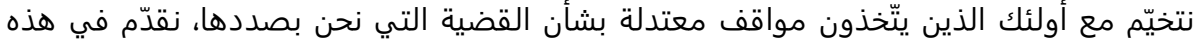

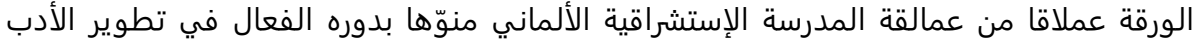
العربي.

الكلمات المفتاحية: الاستشراقية، المستشرقون، اللغات الإسلامية، عملاق، الأدب العربي

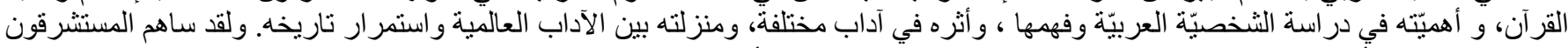
في إحياء التراث الأدبيّ خلال در اسة المخطوطات العربيّة وتحقيق العديد من أمّهات الكتب العربيّة ونرجمة بعض العض هذه الكتب إلى لغات مختلفة، وكتابة 


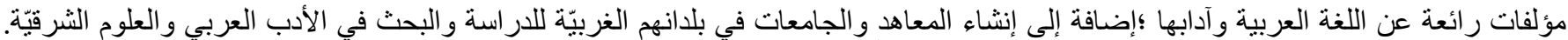

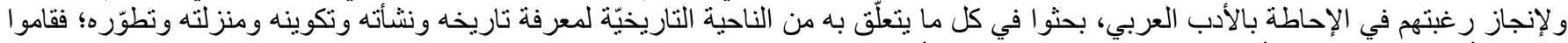

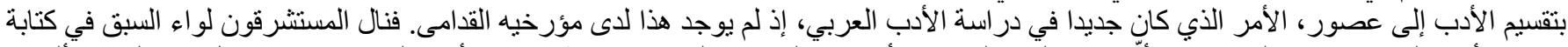

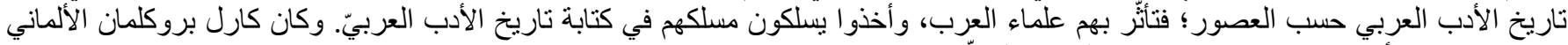
صاحب "كاريخ الأدب العربي" من رواد هذه الحركة المؤثِّة.

الاستثراق: المفهوم، النشأة، والمدارس

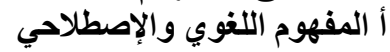

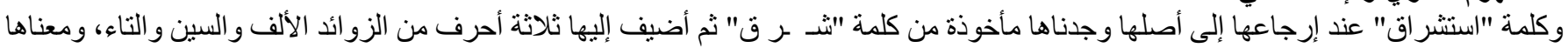
طلب الثرق؛ ولبس طلب الشرق سوى طلب علوم الشرق و آدابه ولغاته و أديانه...

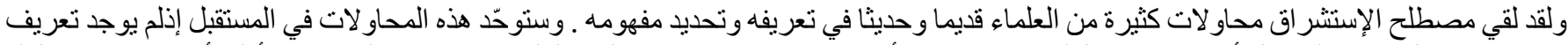

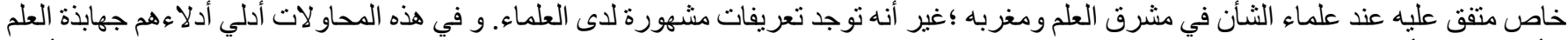

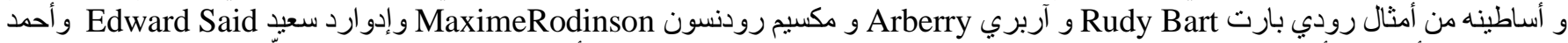

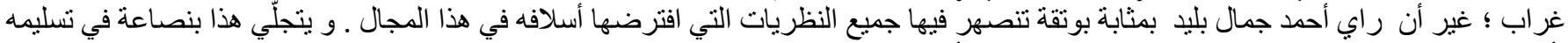

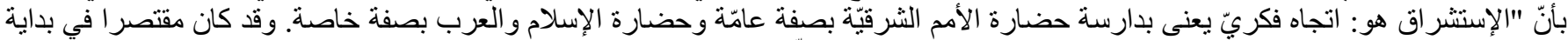

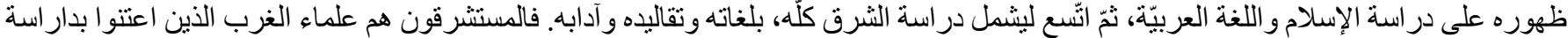

الإسلام و اللغة العربية، ولغات الثرق و أديانه وآدابه.

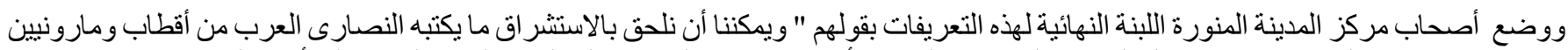

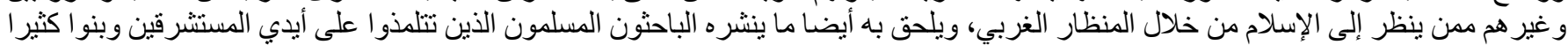

من أفكار المستشرقين2

ب ب - النشأة

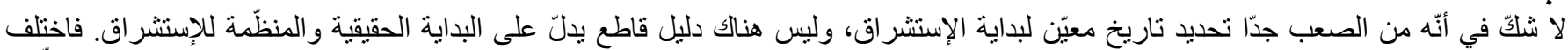

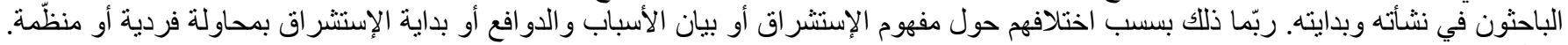

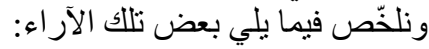

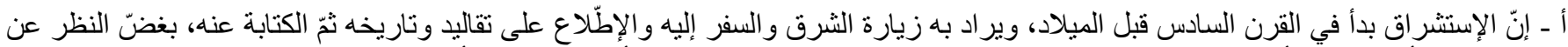

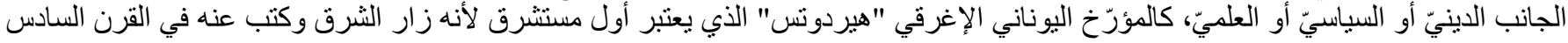

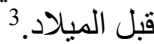

ب ب - إنّ الإستشر اق بدأ في القرن السابع الميلادي في العهد الأموي حيث قام العالم النصر اني يوحنا الدمشقي (686 -749م) بدر اسة الإسلام بقصد التشويه

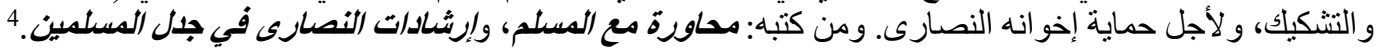

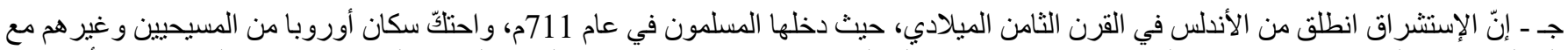

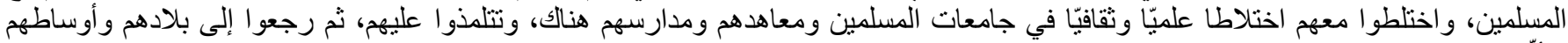
منأترّين بالثقافة الإسلاميّة.

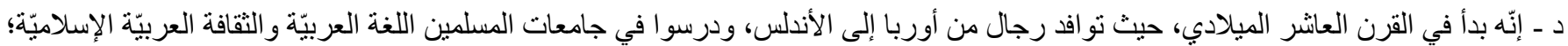

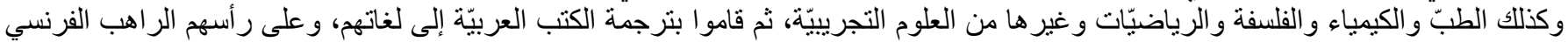

هـ - إنه بدأ في القرن الثاني عشر الميلادي، وذلك حين ترجم الر اهب الإنجليزي "هرمان" القرآن الكريم إلى اللغة اللاتينية عام 1143م، وكما صدر أول قاموس عربي - لاتيني في هذه الفنرة.

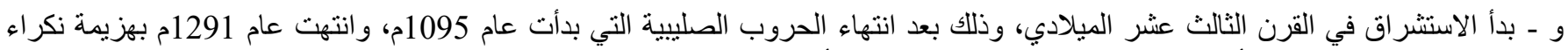
للصلبيين، فتوجهو ا إلى در اسة أسباب غلبة المسلمين وفوز هم، و هكذا بدأ الاستشر اق. 8

ز - بدأ في القرن الر ابع عشر الميلادي، و هو بداية الاستشراق الرسمي، حيث صدر قرار من مجمع فيينا الكنسي عام 1312م بإنشاء عدد من كراسي اللغة العربية في عدة جامعات أوربية.

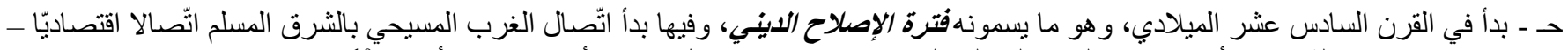

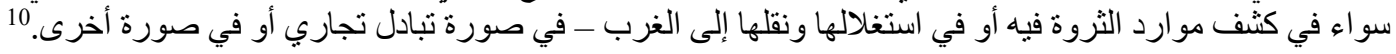

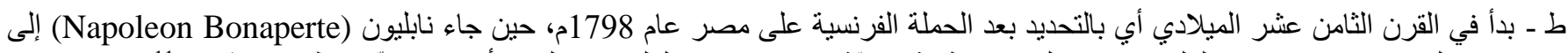
مصر، وحمل معه عددا كبير ا من العلماء، كما حمل معه مطبعة عربيّة ساعدت هؤ لاء العلماء في القيام بأبحاث متعدّدة وطبعها ونشر ها. 


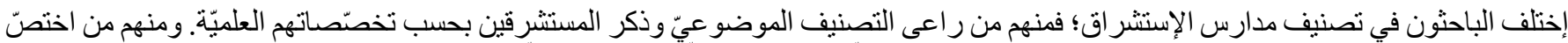

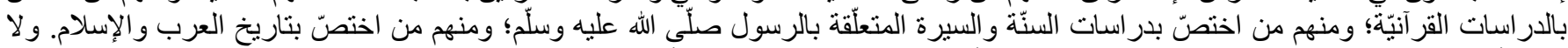

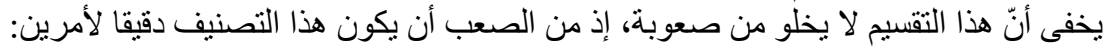
الأول: أنّ معظم المستشرقين قد كتبوا في موضو عات لدات متداخلة، وليس من اليسير على الباحث أن يكون دقيقا في تصنيفه، لصعوبة تحديد اتجاهات المستشرقين بسبب تداخل العلوم الإسلاميّة وتقاربها.

الثاني: من الصعب - وفقا لهذا التصنيف الموضوعيّ - وضع خصائص لكلّ مدرسة من المدارس الإستشر اقية، لأنّ كلّ مدرسة تشتنمل على عدد كبير من المستشرقين يختلفون اختلافا بيّنا في مناهجهم واتجاهاتهم وميولهم، لإختلاف طبائع الثعوب وما نتركه في شعوبها من طبائع وملامح.

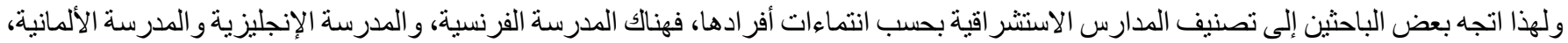

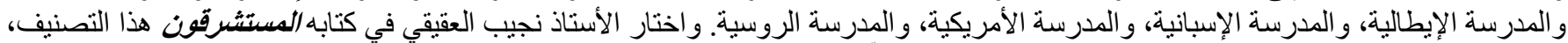

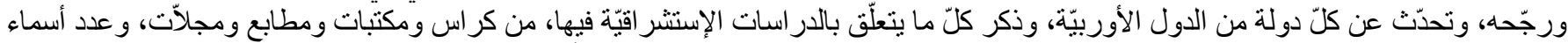

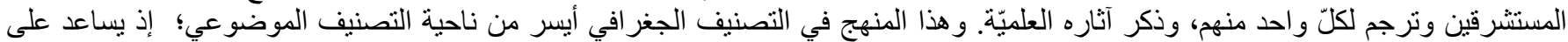

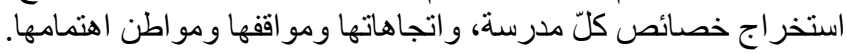

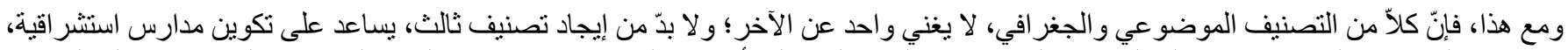

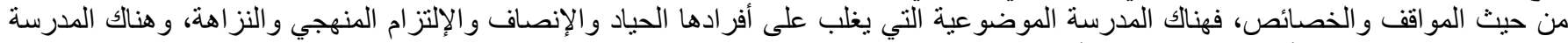

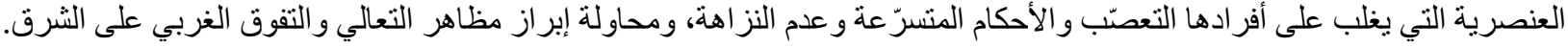

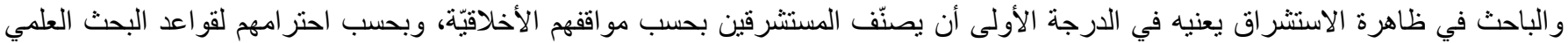

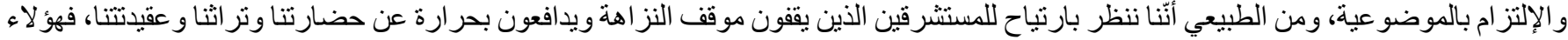
جديرون بالتقدير ، من وجهة نظرنا.

المستشرقون وتاريخ الأدب العربي الإني

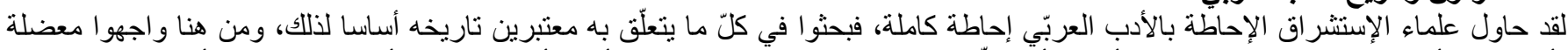

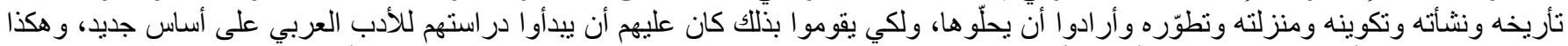

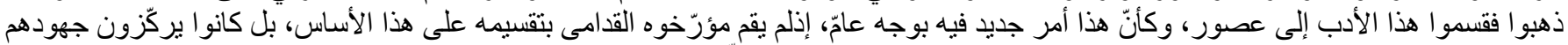

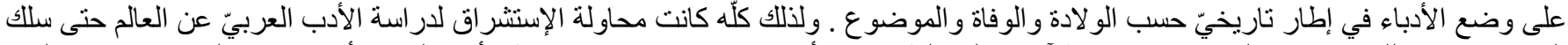

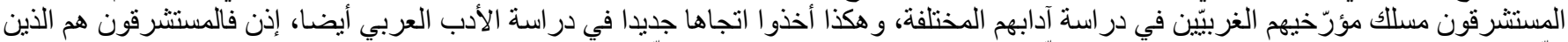

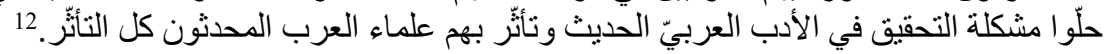

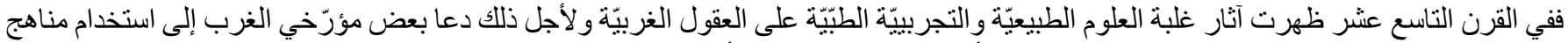

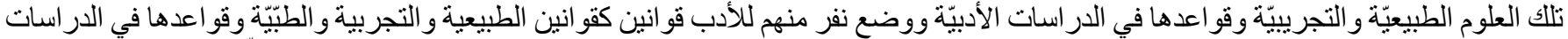

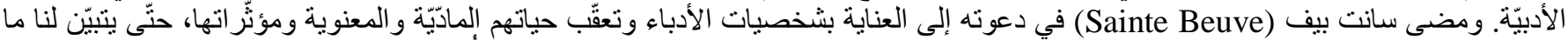

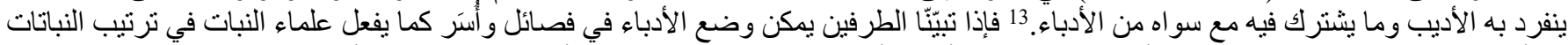

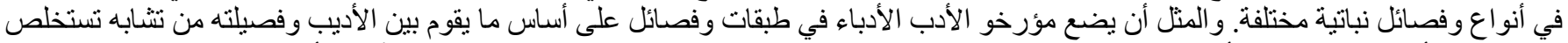

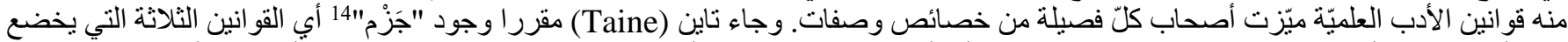

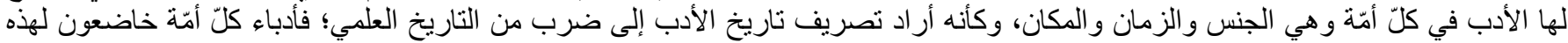

القو انين الثلاثة خضو عا جبريّا ملزما...

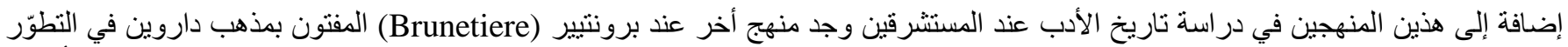

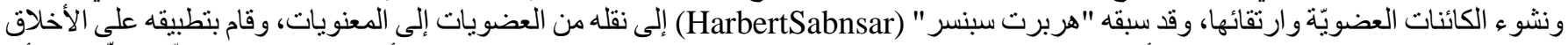

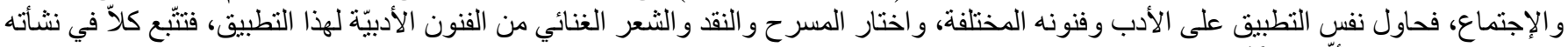
ونموّه وتطورّه وما يتأُّرّ فيه.

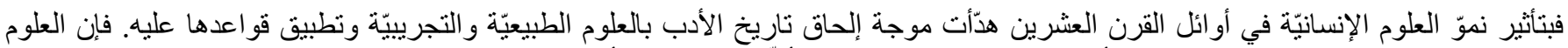

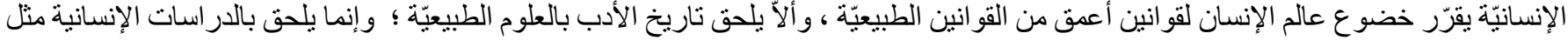

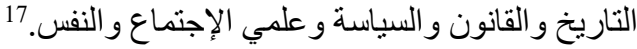

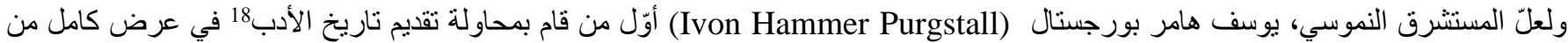

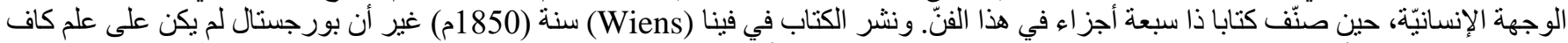

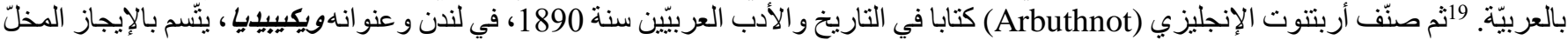

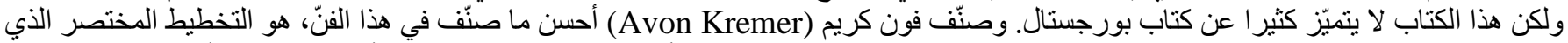

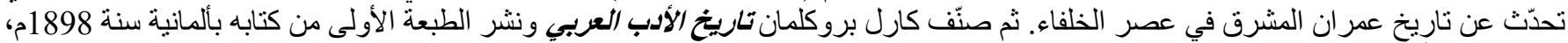

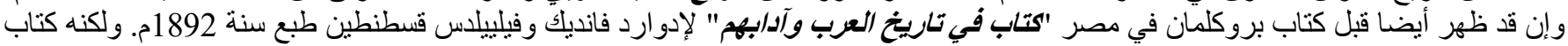




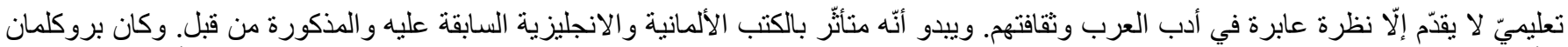

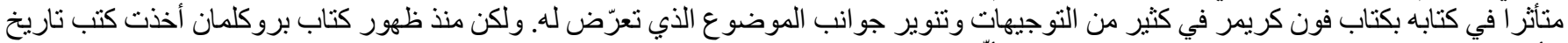

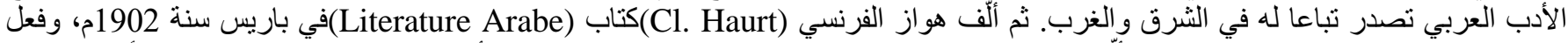

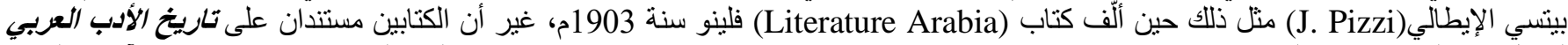

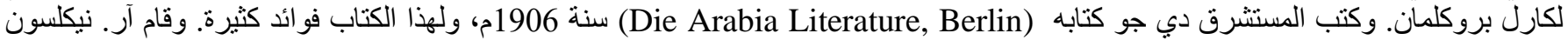
(A Licholas) Renaissance Des Is Islam, Heidelderg)

الفرنسي في هذا الفن.

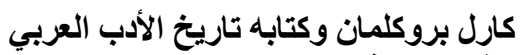

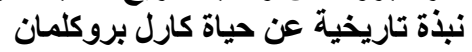

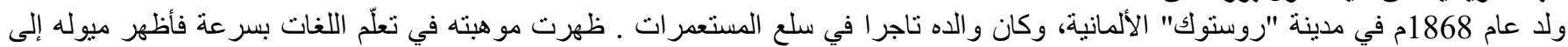

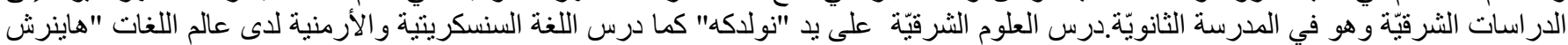

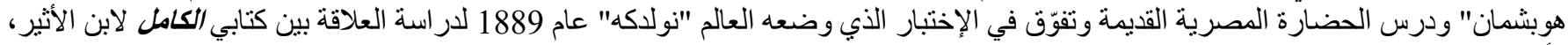

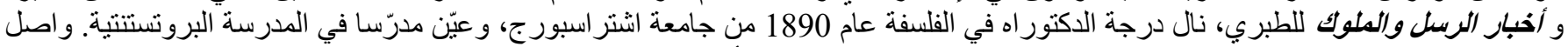

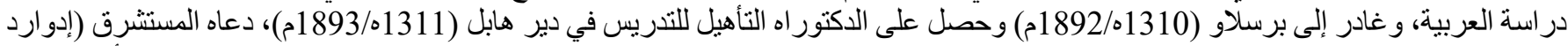

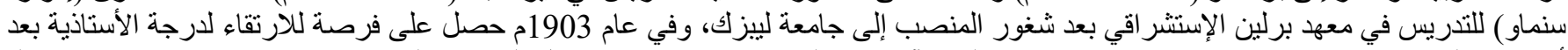

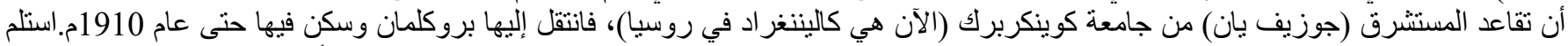

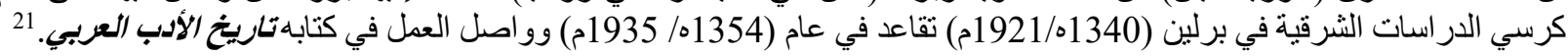

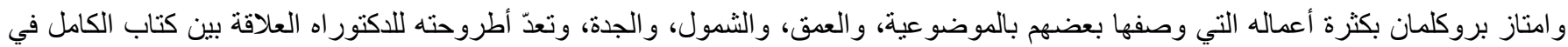

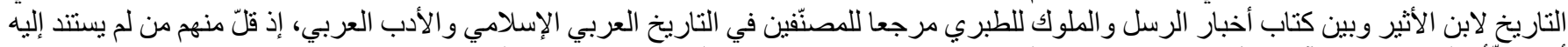

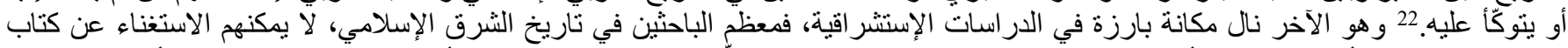

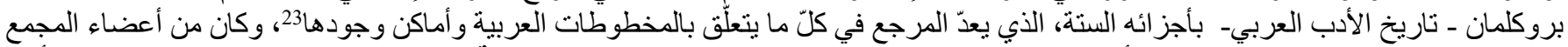

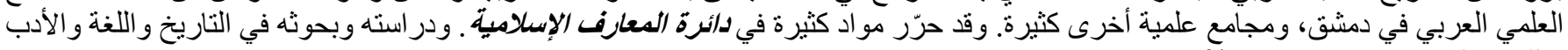
واللغات السامية، غزيرة ووفيرة.

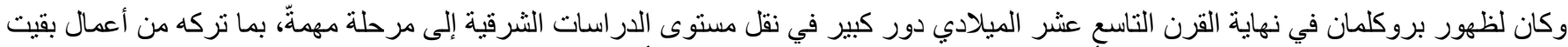

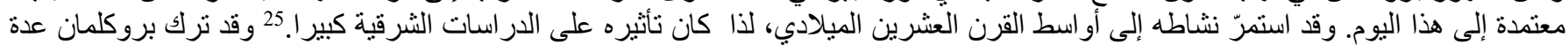

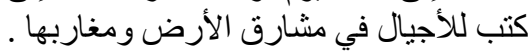

كتاب تاريخ الأدب العربي لبروكلمان

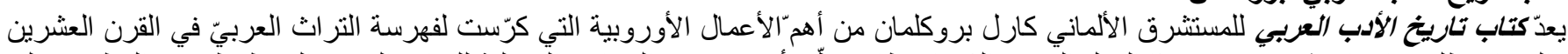

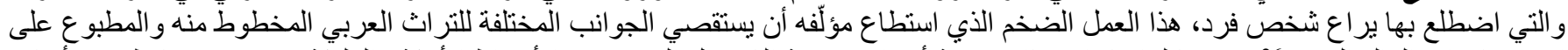

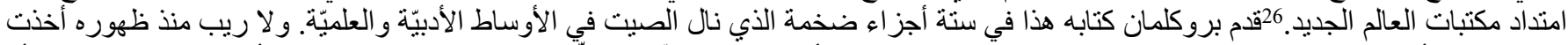

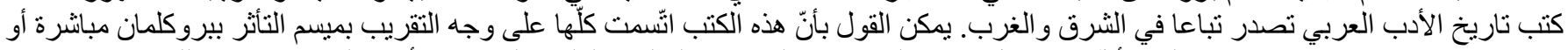

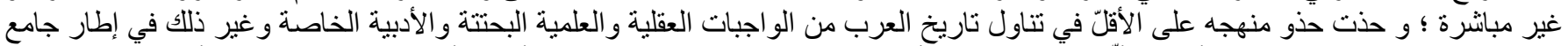

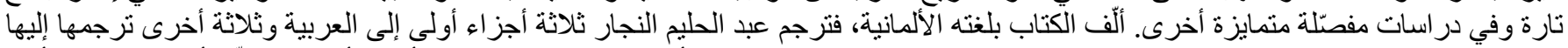

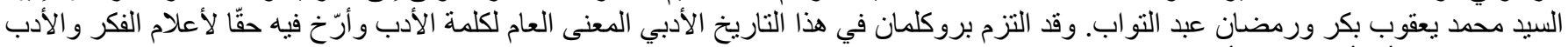

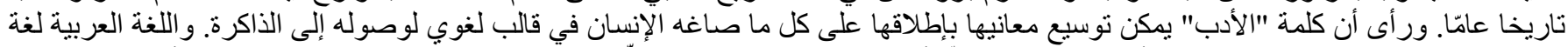

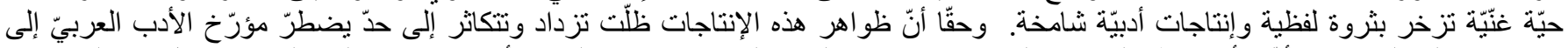

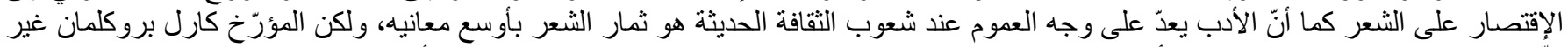

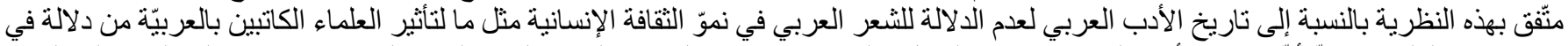

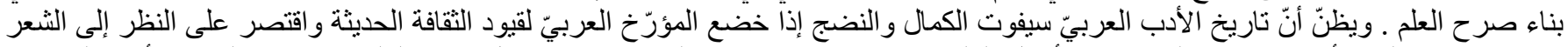

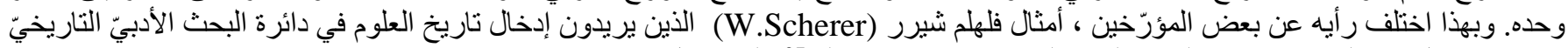

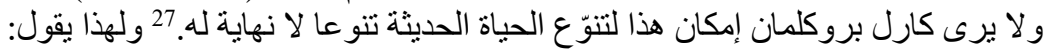

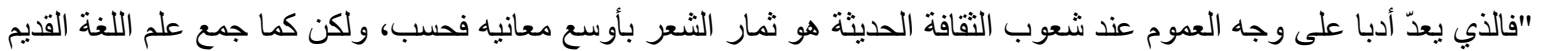

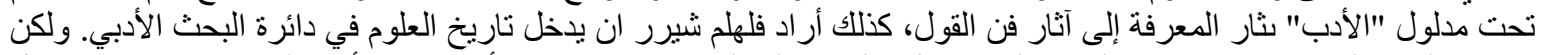

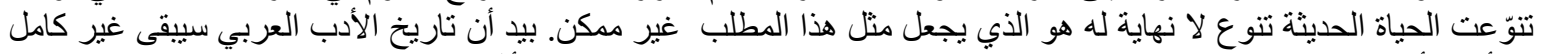

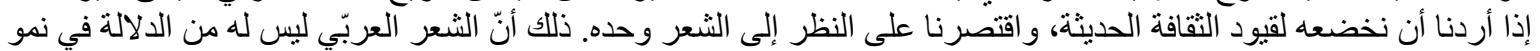

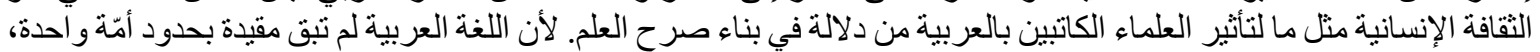

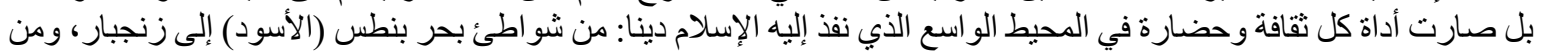
فاس وتمبكتو إلى كثنغر وجزر الملايو ...28 
وكتاب بروكلمان بأجز اءه الستة مقسوم إلى خمسة عصور ففي الجزء الأول تحدّث عن العصر من أوليّة الأمّة العربية (من العصر الجاهلي) إلى ظهور

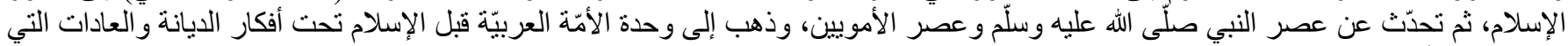

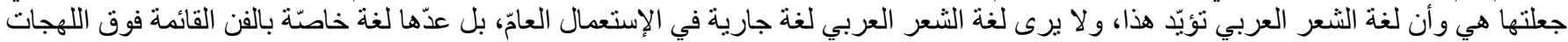
كما عدها جميع المؤرخين.

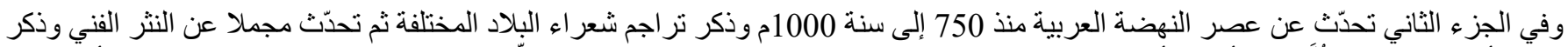

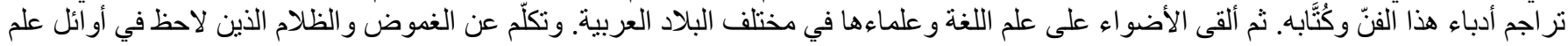
اللغة العربية.

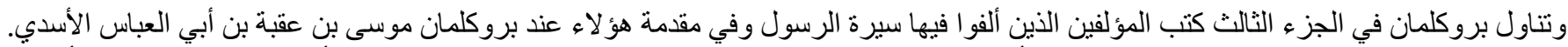

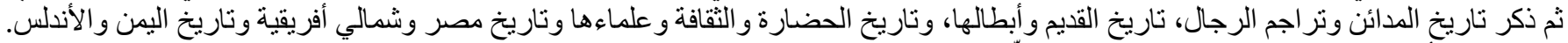

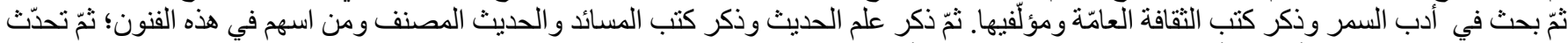

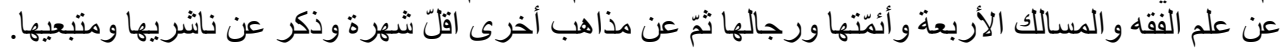

وفي الجزء الر ابع من الكتاب تحدث عن علوم القرءان و وتفسيره و العقائد والتصوف و المنرجمين و الفلسفة والرياضيات وعلم الفلك و التنجيم و الجغر افية

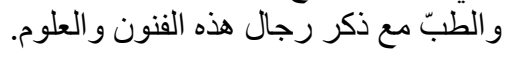

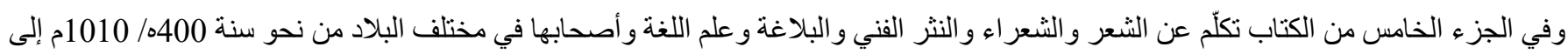

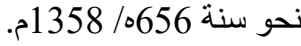

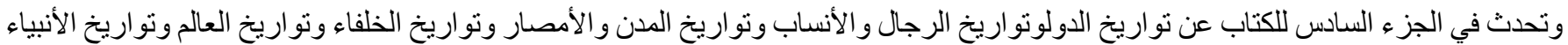

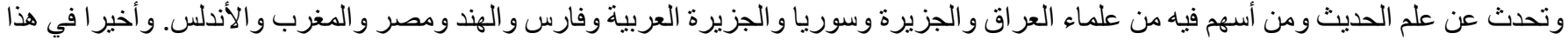
الجزء تحدّث عن علماء فقه الحنفية.

الخاتمة

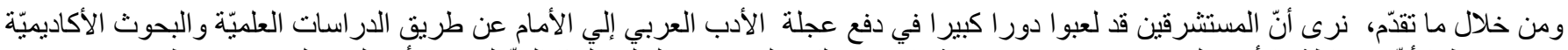

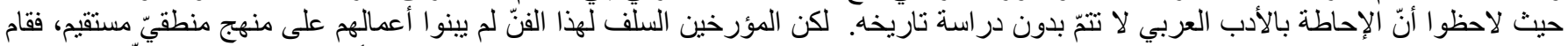

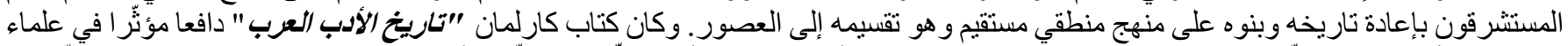

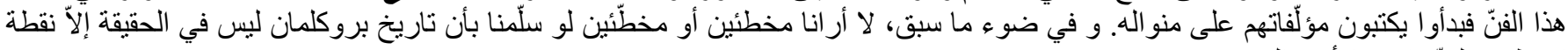

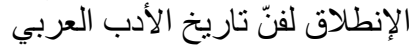

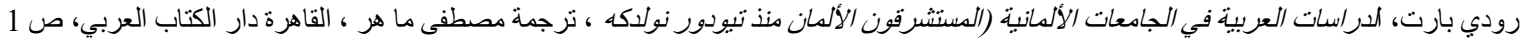

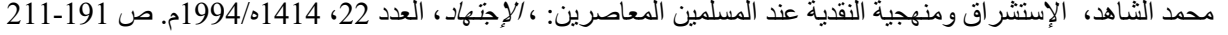

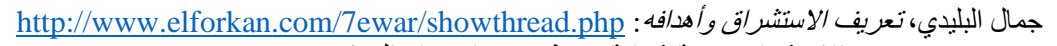

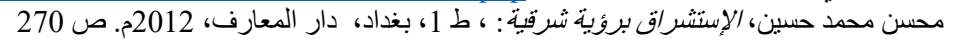

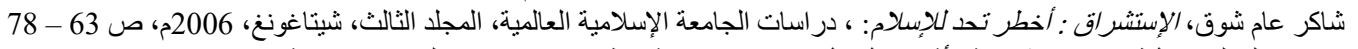

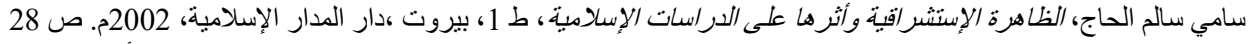

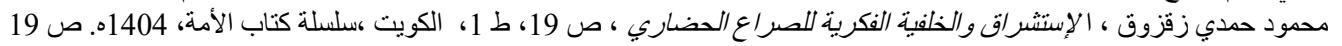

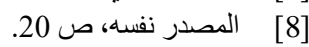

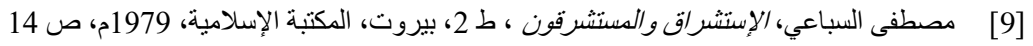

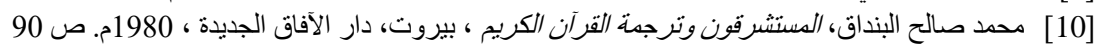

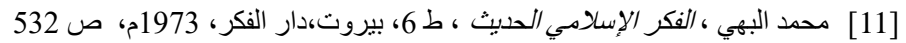

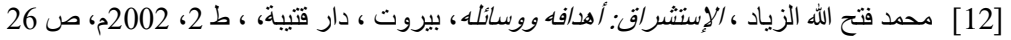

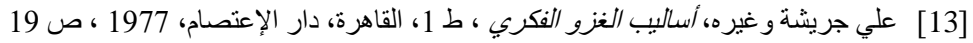

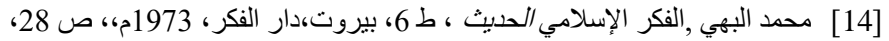

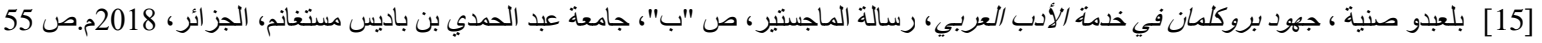

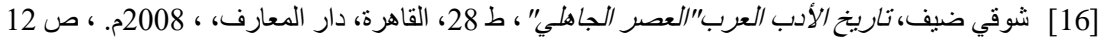

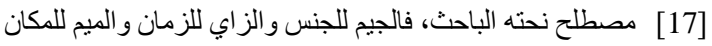

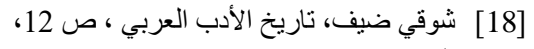
[19] [18] [المصدر نفسه [20] [20]

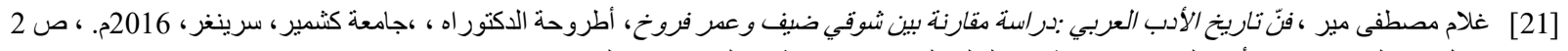

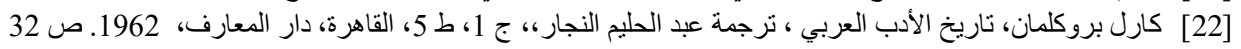

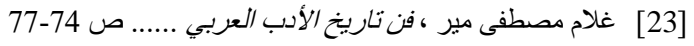

[24] ] عبد الرحمن بدوي، موسوعة المستشرقين ، ط 3 3، بيروت، لبنان، دار العلم للملايين ، 1993م. 
[25] نجيب العقيقي، المستشرقون : دوسوعة في تراث العرب دع تراجم المستشرقين ودراساتهم عنه هنذ ألف عام حتى اليوم ، ، ج 2، ط 5، القاهرة، دار المعارف، 2006م. ص 425

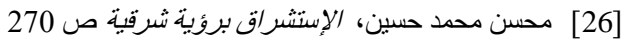

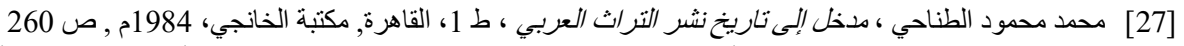

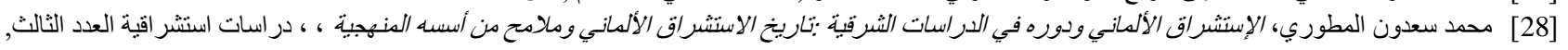
2015م. ، ص 202 